\title{
Polarizability Extraction of Above-Half-Space Transversal Dipole Scatterers Using a Fast Waveguide-Based Approach
}

\author{
Yasser Bigdeli, Mojtaba Dehmollaian, \\ Department of Electrical and Computer Engineering, University of Tehran, Iran \\ *corresponding author, E-mail: yaser.bigdeli@gmail. com
}

\begin{abstract}
We present a semi-analytical method to extract transverse polarizability parameters of an arbitrary bi-anisotropic subwavelength scatterer both in homogeneous medium and placed at the boundary of two simple (homogeneous, isotropic, and linear) media. Using this technique, polarizability parameters of various dielectric and/or metallic scatterers are obtained, effectively. In this method, a scatterer is placed at the middle of a rectangular waveguide which in general is filled by two different simple media in either sides of the scatterer. The waveguide is designed so that the two TE10 and TE01 fundamental modes are propagating in a given frequency band. All 16 transverse polarizabilities are fast obtained having 16 different generalized scattering parameters (S-parameters). The S-parameters are associated with excitations at two different ports of the waveguide and the two different modes (TE10 and TE01). Comparing to existing polarizability extraction methods, the presented waveguide method is easy to implement, fast and almost accurate. In order to validate the method, we present three examples including omega particle and magneto-dielectric sphere in free-space and an electric resonance particle, placed on top of a dielectric half-space.
\end{abstract}

\section{Introduction}

Metamaterials composed of small inclusions positioned near each other, provide exotic electromagnetic (EM) properties that do not exist naturally [1-12]. Inclusions (meta-atoms) are electrically small scatterers that essentially offer interesting macroscopic parameters based on their geometrical and physical properties. Effectively, (dipole) scatterers are described by their polarizability parameters $(\overline{\bar{\alpha}})$ which are physical parameters that depend on frequency and particle shape and material. They show how a single scatterer responds to an external EM field. Having known different polarizabilities, realization of a wide range of metasurfaces and metamaterials could be made possible.

There are various analytical and numerical methods to calculate polarizability tensors of scatterers. Analytical methods are limited to some simple structures. Indeed, small modifications to the scatterer structure result in a different analytical model [3]. For example, analytical models for a wire dipole [4], wire chiral [5], wire omega [6], Tellegen- omega, and a moving-chiral [7] scatterers have been reported before.

On the other hand, numerical methods are mainly based on evaluating the scattered fields of the subwavelength scatterer under several test illuminations. In [8], polarizability tensors of an arbitrary scatterer in free-space are extracted by plane-wave illuminations and far-field measurements. In [3], formulation of [8] is extended to retrieve polarizabilities of an arbitrary scatterer, placed on top of a half-space. In that formulation, dipole moments are evaluated directly by numerically integrating induced currents in/on scatterer at different illuminations. This approach is taken instead of the method proposed in [8], calculating the scattered fields at two different far-field sample points.

In [9] and [10], the reflection and transmission coefficients of an array of similar scatterers are employed. Array effect is accounted for by analytically computing the "interaction coefficients". Therefore, having known array effect, scatterer polarizability parameters can be expressed in terms of measured scattering parameters. The method has an advantage of simulating a relatively small bounded medium compared to the large computation domain of [3]. It requires simpler simulation procedure by introducing periodic boundary condition and deals with a smaller computational domain. However, this method is applied to arrays in a homogeneous medium. Basically, analytical derivation of interaction fields for arrays placed inside a homogeneous medium is straight forward [1]. In contrast, in practice metasurfaces are mainly fabricated on a dielectric slab or placed on top of a half-space. In that cases, computation of interaction coefficients is not easy and to the best of our knowledge no closed-form analytical formulation has been reported so far.

In [2], a similar array-based method is proposed in which interaction coefficients are extracted by a calibration procedure. Indeed, the calibration is done by a particle with analytically known polarizabilities, like a dielectric sphere. Then, using a similar process addressed in [9] and [10], polarizabilities are expressed in terms of the scattering parameters and interaction coefficients. Formulations in [2] are presented for a scatterer inside a homogeneous medium. It may be challenging to find a calibration procedure for the case where a scatterer is placed on a half-space dielectric. Because in that case, the polarizabilities depends on the calibrating particle [14]. 
To solve the issues of [9] and [10] mentioned above, here we present a general formulation based on a waveguide scattering parameter calculation. In [11], a rectangular waveguide (WG) approach is proposed for a special case of an artificial Tellegen particle. Here, we extend the rectangular WG method of [11] and propose general and explicit formulations to retrieve transversal polarizabilities of any arbitrary small scatterer. In general, we consider a scatterer to be placed on a simple (homogeneous, isotropic, and linear) dielectric half-space.

The paper is organized as follows. First, in Section 2, initial idea is presented. We present and discuss waveguide extraction method formulations in Section 3. Section 4 verifies the formulations by different numerical examples. Concluding remarks are given in Section 5.

\section{Initial Idea}

As shown in Fig. 1, an arbitrary small scatterer is placed at the center of a WG which is half filled by a dielectric medium. Rectangular WG is excited by two wave-ports at its two ends. The WG is designed so that TE10 and TE01 modes are propagating in a given desired frequency band. Transverse field components of these two modes received at the ports are indeed orthogonal to each other. Therefore, they together can carry co- and cross-polarizations of scattered fields, generated by the particle.

All transverse polarizability parameters are extracted using four incidences; two modes (TE10 and TE01) excited at two ports. Each incidence provides four scattering (S) parameters in two polarizations (co and cross) received at two ports. This provides 16 equations, used to find 16 unknown transverse polarizabilities. Our method can treat metasurfaces that have only transversal (not normal) polarizabilities.

In our approach, a dipole scatterer is first modeled by electric $(\bar{p})$ and magnetic $(\bar{m})$ dipole moments, given in terms of S-parameters measured at different ports and different modes. Then, polarizability parameters $(\overline{\bar{\alpha}})$ are derived in terms of the dipole moments.

Indeed, an electric and/or magnetic dipole inside a WG excites multiple TE and/or TM modes. However, we can effectively approximate fields inside the waveguide by dominant TE10 and TE01 mode fields and neglect higherorder modes. It is noted that the first four higher-order modes, namely TM11, TE11, TE20, and TE02 have zero field values at the WG center and they may not even get excited.

Importantly, in the case of a half-filled WG, we note that modes cut-off frequencies decreases and generally, some higher-order modes may become propagating in the dielectric loaded region. As long as amplitude of these higher-order modes could be still assumed negligible, our proposed method works properly and reasonable accuracy could be obtained. This is the case for substrates with relatively low permittivity values.

Thus, in applying the boundary conditions (BCs) at $z=0$ (See Fig.1), only two first fundamental modes (TE10 and TE01) are assumed to be present. This assumption does not

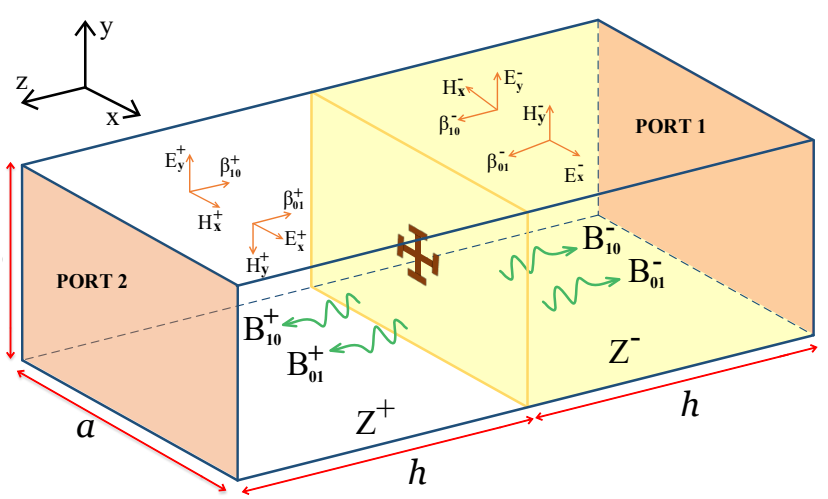

Figure 1: Waveguide simulation structure. In general case, the scatterer is placed in the center of the waveguide at $z=0$. The region $z<0$ and $z>0$ have characteristic impedance $Z^{-}$and $Z^{+}$, respectively.

make considerable discrepancies and accurate results are yet obtained.

In fact, EM fields at $z=0$ (in Fig. 1) should have relatively smooth phase and amplitude transversal variations as that of TE10 and TE01 modes in order to have a decent approximation. Thus, in presented WG-based method, WG sides to particle length ratio is set large enough (as a rule of thumb this ratio is about 5). Also, ports are placed far enough (about 2 wavelength) at $z= \pm h$ to extinguish evanescent modes effects.

\section{Proposed WG-based Method Formulations}

A sub-wavelength bi-anisotropic scatterer can be modeled by induced electric $(\bar{p})$ and magnetic $(\bar{m})$ dipole moments that are related to local electric $\left(\bar{E}_{\text {loc }}\right)$ and magnetic $\left(\bar{H}_{\text {loc }}\right)$ fields through polarizability tensors as follow [1],

$$
\begin{gathered}
\bar{p}=\overline{\bar{\alpha}}_{e e} \cdot \bar{E}_{\mathrm{loc}}+\overline{\bar{\alpha}}_{e m} \cdot \bar{H}_{\mathrm{loc}}, \\
\bar{m}=\overline{\bar{\alpha}}_{m e} \cdot \bar{E}_{\mathrm{loc}}+\overline{\bar{\alpha}}_{m m} \cdot \bar{H}_{\mathrm{loc}},
\end{gathered}
$$

where $\overline{\bar{\alpha}}_{\mathrm{ee}}, \overline{\bar{\alpha}}_{\mathrm{em}}, \overline{\bar{\alpha}}_{\mathrm{me}}$, and $\overline{\bar{\alpha}}_{\mathrm{mm}}$ are electric, magnetic, magnetoelectric, and electromagnetic polarizability tensors, respectively. Here in general, polarizability tensors are $3 \times 3$ tensors and therefore, there are 36 unknowns to be retrieved.

In (1), inside the WG structure, local fields are the incident fields from the WG ports. Considering TE10 and TE01 illuminations, both $x$ and $y$ directed electric and magnetic field components are present. Therefore, in the WG simulation setup, we can effectively determine all transverse polarizability components (16 unknowns) through one simulation run. It should be noted that under TE10 and TE01 illuminations, longitudinal magnetic field $\left(H_{z}\right)$ is the only non-zero normal field component. However, this component is zero in the scatterer place (i.e., in the middle of the WG at $x=a / 2, y=b / 2$, and $z=0)$. Therefore, it has no contribution in the reflected and transmitted fields.

Indeed, in the free-space medium full polarizability tensors (36 unknowns) can be extracted by properly rotating the scatterer, which requires 3 simulation runs. However, in the half-filled medium normal components of polarizabilities (remained 20 parameters) cannot be determined due to the 
variation on normal dipole moments with respect to the dielectric interface. Therefore, our method can treat abovehalf-space scatterers that have only transversal polarizabilities.

By replacing the scatterer by equivalent induced electric and magnetic dipole moments, the problem transforms in to calculation of radiated fields due to electric and magnetic point sources positioned at the WG center. This is given mathematically by the following $\mathrm{BCs}$,

$$
\begin{aligned}
& \hat{z} \times \Delta \bar{H}=j \omega \bar{p} \delta(x-a / 2) \delta(y-b / 2), \\
& \Delta \bar{E} \times \hat{z}=j \omega \bar{m} \delta(x-a / 2) \delta(y-b / 2),
\end{aligned}
$$

at $z=0$, where $\Delta \bar{E}$ and $\Delta \bar{H}$ are fields differences at $z=0$ [e.g., $\left.\Delta \bar{E}=\bar{E}\left(z=0^{+}\right)-\bar{E}\left(z=0^{-}\right)\right], \omega$ is angular frequency and $\delta$ is the well-known delta function.

Each one of TE10 and TE01 mode incidences from the WG two ports provide orthogonal field components. This leads to two separate sets of equations. According to Fig. 1, TE10 incidence include $y$-directed electric field $E_{y i n c}^{ \pm}=$ $E_{0} \sin (\pi x / a)$ and $x$-directed magnetic field $H_{x i n c}^{ \pm}=$ $\pm E_{\text {yinc }}^{ \pm} / Z_{10}^{ \pm}$. Here, in our convention which is also shown in Fig. 1., \pm signs in all parameters, refer to their regions; + and - are used for the regions $z>0$ and $z<0$, respectively. For example $E_{y}^{-}$is the electric field in region $\mathrm{z}<0$ while $E_{y}^{+}$is that in $\mathrm{z}>0$. Using (1) and considering TE10 incidence from ports 1 and 2, one set of equations is as follows.

$$
\begin{gathered}
p_{i}^{(1)}=\left(\alpha_{e e}^{i y}-\alpha_{e m}^{i x} / Z_{10}^{-}\right) E_{0}, p_{i}^{(2)}=\left(\alpha_{e e}^{i y}+\alpha_{e m}^{i x} / Z_{10}^{+}\right) E_{0}, \\
m_{i}^{(1)}=\left(\alpha_{m e}^{i y}+\alpha_{m m}^{i x} / Z_{10}^{+}\right) E_{0}, m_{i}^{(2)}=\left(\alpha_{m e}^{i y} 0 \alpha_{m m}^{i x} / Z_{10}^{-}\right) E_{0},
\end{gathered}
$$

where superscripts (1) and (2) indicate that the dipole moment is excited by an incident wave from port 1 and 2, respectively. The waves from port 1 and 2 are forward $+z$ and $-z$ propagating waves, respectively. In (3), $i=x, y$ and $Z_{10}^{ \pm}=\omega \mu / \beta_{10}^{ \pm}$and $\beta_{10}^{ \pm}=\left[k_{0}^{2} \varepsilon_{r}^{ \pm}-(\pi / a)^{2}\right]^{1 / 2}$ are the TE10 wave impedance and propagation constants at each of the two \pm regions ( $z>0$ and $z<0$ regions), respectively. Here, the incident electric field amplitude is assumed to be $E_{0}$.

Solving equations set (3), one may retrieve polarizabilities in terms of dipole moments as follow,

$\alpha_{e e}^{i y}=\frac{z_{10}^{-} p_{i}^{(1)}+Z_{10}^{+} p_{i}^{(2)}}{\left(z_{10}^{+}+Z_{10}^{-}\right) E_{0}}, \alpha_{m m}^{i x}=\frac{-m_{i}^{(1)}+m_{i}^{(2)}}{\left(1 / Z_{10}^{+}+1 / Z_{10}^{-}\right) E_{0}}$,

$\alpha_{m e}^{i y}=\frac{Z_{10}^{-} m_{i}^{(1)}+Z_{T E 10}^{+} m_{i}^{(2)}}{\left(Z_{10}^{+}+Z_{10}^{-}\right) E_{0}}, \alpha_{e m}^{i x}=\frac{-p_{i}^{(1)}+p_{i}^{(2)}}{\left(1 / Z_{10}^{+}+1 / Z_{10}^{-}\right) E_{0}}$.

Similarly, using (1) and assuming TE01 incidence which includes $x$-directed electric field $E_{x i n c}^{ \pm}=E_{0} \sin (\pi y / b)$ and $y$-directed magnetic field $H_{\text {yinc }}^{ \pm}=\mp E_{\text {xinc }}^{ \pm} / Z_{01}^{ \pm}$, two other equations are obtained as follow.

$p_{i}^{(1)}=\left(\alpha_{e e}^{i x}+\alpha_{e m}^{i y} / Z_{01}^{-}\right) E_{0}, p_{i}^{(2)}=\left(\alpha_{e e}^{i x}-\alpha_{e m}^{i y} / Z_{01}^{+}\right) E_{0}$,

$m_{i}^{(1)}=\left(\alpha_{m e}^{i x}-\alpha_{m m}^{i y} / Z_{01}^{+}\right) E_{0}, m_{i}^{(2)}=\left(\alpha_{m e}^{i x}+\alpha_{m m}^{i y} / Z_{01}^{-}\right) E_{0}$,

where $Z_{01}^{ \pm}=\omega \mu / \beta_{01}^{ \pm}$and $\beta_{01}^{ \pm}=\left[k_{0}^{2} \varepsilon_{r}^{ \pm}-(\pi / b)^{2}\right]^{1 / 2}$ are the TE01 wave impedance and propagation constant, respectively. Using (5), other eight transverse polarizability parameters are obtained in terms of related dipole moments as follow.

$$
\begin{aligned}
& \alpha_{e e}^{i x}=\frac{z_{01}^{-} p_{i}^{(1)}+z_{01}^{+} p_{i}^{(2)}}{\left(z_{01}^{+}+z_{01}^{-}\right) E_{0}}, \alpha_{m m}^{i y}=\frac{m_{i}^{(1)}-m_{i}^{(2)}}{\left(1 / z_{01}^{+}+1 / z_{01}^{-}\right) E_{0}}, \\
& \alpha_{m e}^{i x}=\frac{z_{01}^{-} m_{i}^{(1)}+z_{01}^{+} m_{i}^{(2)}}{\left(z_{01}^{+}+z_{01}^{-}\right) E_{0}}, \alpha_{e m}^{i y}=\frac{p_{i}^{(1)}-p_{i}^{(2)}}{\left(1 / z_{01}^{+}+1 / z_{01}^{-}\right) E_{0}},
\end{aligned}
$$

Next, we express dipole moments in terms of reflected and transmitted fields. Therefore, we use boundary conditions (2) for each one of the incidences. The scattered fields in the two regions are assumed to be [13]

$$
\begin{gathered}
\bar{E}_{S}^{ \pm}=B_{01}^{ \pm} \sin \left(\frac{\pi y}{b}\right) \hat{x}+B_{10}^{ \pm} \sin \left(\frac{\pi x}{a}\right) \hat{y}, \\
\bar{H}_{S}^{ \pm}=\left[\mp \frac{B_{10}^{ \pm}}{Z_{10}^{ \pm}} \sin \left(\frac{\pi x}{a}\right) \hat{x} \pm \frac{B_{01}^{ \pm}}{z_{01}^{ \pm}} \sin \left(\frac{\pi y}{b}\right) \hat{y}\right],
\end{gathered}
$$

in which the upper and lower signs correspond to the waves in \pm regions and propagating along $+z$ and $-z$ directions, respectively as mentioned before. According to Fig. 1, for example, $\mathrm{B}_{10}^{+}$is the amplitude of scattered TE10 field propagating along $+z$ direction in region $\mathrm{z}>0$.

To completely satisfy BCs (2), all higher-order modes should have been included in (7) to form the scattered fields, accurately [13]. As discussed in Section 2, here we assume that only TE10 and TE01 modes are mainly contributing and we neglect higher order modes.

In Eq. (2), first, the delta function $(\delta)$ is expanded in to a series of orthogonal basis functions. Next, we take inner product of both sides of (2) with associated basis functions. This way, we retrieve a desired mode field amplitude. This is explained in Appendix I. Basically, by using the scattered fields given in (7) and applying BCs (2) at the scatterer position and considering both incidences (i.e., incidences from port 1 and port 2), we can solve for the induced dipole moments in terms of scattered fields coefficients (See Appendix I).

Dipole moments evaluated from both TE10 and TE01 incidences are as follow.

$p_{y}^{(1)}=\frac{a b}{2 j \omega}\left(-\frac{B_{10}^{+}}{Z_{10}^{+}}-\frac{B_{10}^{-}}{Z_{10}^{-}}+\frac{E_{0}}{Z_{10}^{+}}\right), p_{y}^{(2)}=\frac{a b}{2 j \omega}\left(-\frac{B_{10}^{+}}{Z_{10}^{+}}-\frac{B_{10}^{-}}{Z_{10}^{-}}+\frac{E_{0}}{Z_{10}^{-}}\right)$, $m_{x}^{(1)}=\frac{a b}{2 j \omega}\left(B_{10}^{+}-B_{10}^{-}-E_{0}\right), m_{x}^{(2)}=\frac{a b}{2 j \omega}\left(B_{10}^{+}-B_{10}^{-}+E_{0}\right)$, $p_{x}^{(1),(2)}=-\frac{a b}{2 j \omega}\left(\frac{B_{01}^{+}}{z_{01}^{+}}+\frac{B_{01}^{-}}{z_{01}^{-}}\right), m_{y}^{(1),(2)}=-\frac{a b}{2 j \omega}\left(B_{01}^{+}-B_{01}^{-}\right)$.

where each one of the scattered field coefficients ( $B$ 's) are proportional to a particular S-parameter.

It is noted that for the half-filled WG, the port impedances are different. Therefore, in this case we need to use the generalized scattering parameters [15]. Therefore, in scattering parameters definition, each one of the scattered and incident waves should be normalized to a relevant characteristic impedance, associated with the mode and region. Therefore,

$$
B_{m}^{r}=\sqrt{Z_{m}^{r}} S_{m n}^{r p} E_{0} / \sqrt{Z_{n}^{p}}
$$


where $S_{m n}^{r p}$ is the S-parameter determined by $r$ and $p=1$ or 2 (mode number) and $m$ and $n=1$ or 2 (port number). For example, $S_{12}^{21}$ is the S-parameter when incident mode is 1 (TE10) and received mode is 2 (TE01) and transmitting port is 2 and receiving port is 1. Equation (9) provides field amplitude $B_{m}^{r}$ of mode $r$ at port $m$ (in region $m$ ) due to the excitation at port $n$ (region $n$ ) by mode $p$. For example, for TE10 excitation at port $1, \mathrm{~B}_{01}^{+}$is equal to forward TE01 scattered field amplitude and therefore is proportional to $\mathrm{S}$ - parameter $S_{21}^{21}$. Clearly, in (9), $\mathrm{Z}_{\mathrm{m}}^{\mathrm{r}}$ is the characteristic impedance of mode $r$ at port $m$.

Using this compact notation for the S-parameters and employing (9), (8), and (4), we find relations for polarizabilities in terms of scattering parameters as given by (10.a) -(10.h), at the bottom of this page. Using a similar procedure for TE01 incidences, by employing retrieved dipole moments in (6) and (8) and (9), related polarizabilities are retrieved in terms of S-parameters as given by (11.a) - (11.h) in the following.

$$
\begin{aligned}
& \alpha_{e e}^{y y}=-\frac{a b}{2 j \omega} \frac{S_{11}^{11}+S_{22}^{11}+\sqrt{Z_{10}^{+} / Z_{10}^{-}} S_{12}^{11}+\sqrt{Z_{10}^{-} / Z_{10}^{+}} S_{21}^{11}-2}{\left(Z_{10}^{+}+Z_{10}^{-}\right)} \\
& \alpha_{e e}^{x y}=-\frac{a b}{2 j \omega} \frac{\sqrt{Z_{10}^{-} / Z_{01}^{+}} S_{21}^{21}+\sqrt{Z_{10}^{-} / Z_{01}^{-}} S_{11}^{21}+\sqrt{Z_{10}^{+} / Z_{01}^{+}} S_{22}^{21}+\sqrt{Z_{10}^{+} / Z_{01}^{-}} S_{12}^{21}}{\left(Z_{10}^{+}+Z_{10}^{-}\right)} \\
& \alpha_{m m}^{x x}=\frac{a b}{2 j \omega} \frac{S_{11}^{11}+S_{22}^{11}-\sqrt{Z_{10}^{+} / Z_{10}^{-}} S_{21}^{11}-\sqrt{Z_{10}^{-} / Z_{10}^{+}} S_{12}^{11}-2}{\left(1 / Z_{10}^{+}+1 / Z_{10}^{-}\right)} \\
& \alpha_{m m}^{y x}=\frac{a b}{2 j \omega} \frac{\sqrt{Z_{01}^{-} / Z_{10}^{-}} S_{11}^{21}+\sqrt{Z_{01}^{+} / Z_{10}^{+}} S_{22}^{21}-\sqrt{Z_{01}^{+} / Z_{10}^{-}} S_{21}^{21}-\sqrt{Z_{01}^{-} / Z_{10}^{+}} S_{12}^{21}}{\left(1 / Z_{10}^{+}+1 / Z_{10}^{-}\right)} \\
& \alpha_{m e}^{x y}=\frac{a b}{2 j \omega} \frac{\sqrt{Z_{10}^{-} Z_{10}^{+}} S_{21}^{11}-Z_{10}^{-} S_{11}^{11}+Z_{10}^{+} S_{22}^{11}-\sqrt{Z_{10}^{-} Z_{10}^{+}} S_{12}^{11}+Z_{10}^{+}-Z_{10}^{-}}{\left(Z_{10}^{+}+Z_{10}^{-}\right)} \\
& \alpha_{m e}^{y y}=\frac{a b}{2 j \omega} \frac{\sqrt{Z_{10}^{-} Z_{01}^{-}} S_{11}^{21}-\sqrt{Z_{10}^{-} Z_{01}^{+}} S_{21}^{21}+\sqrt{Z_{10}^{+} Z_{01}^{-}} S_{12}^{21}-\sqrt{Z_{10}^{+} Z_{01}^{+}} S_{22}^{21}}{\left(Z_{10}^{+}+Z_{10}^{-}\right)} \\
& \alpha_{e m}^{y x}=\frac{a b}{2 j \omega} \frac{S_{21}^{11} / \sqrt{Z_{10}^{-} Z_{10}^{+}}+S_{11}^{11} / Z_{10}^{-}-S_{22}^{11} / Z_{10}^{+}-S_{12}^{11} / \sqrt{Z_{10}^{+} Z_{10}^{-}}-1 / Z_{10}^{-}+1 / Z_{10}^{+}}{\left(1 / Z_{10}^{+}+1 / Z_{10}^{-}\right)} \\
& \alpha_{e m}^{\chi x}=\frac{a b}{2 j \omega} \frac{S_{21}^{21} / \sqrt{Z_{01}^{+} Z_{10}^{-}}+S_{11}^{21} / \sqrt{Z_{01}^{-} Z_{10}^{-}}-S_{22}^{21} / \sqrt{Z_{01}^{+} Z_{10}^{+}}-S_{12}^{21} / \sqrt{Z_{10}^{+} Z_{01}^{-}}}{\left(1 / Z_{10}^{+}+1 / Z_{10}^{-}\right)} \\
& \alpha_{e e}^{x x}=-\frac{a b}{2 j \omega} \frac{S_{11}^{22}+S_{22}^{22}+\sqrt{Z_{01}^{-} / Z_{01}^{+}} S_{12}^{22}+\sqrt{Z_{01}^{+} / Z_{01}^{-}} S_{21}^{22}-2}{\left(Z_{01}^{+}+Z_{01}^{-}\right)} \\
& \alpha_{e e}^{y x}=-\frac{a b}{2 j \omega} \frac{\left.\sqrt{Z_{01}^{-} / Z_{10}^{+}} S_{21}^{12}+\sqrt{Z_{01}^{-} / Z_{10}^{-}} S_{11}^{12}+\sqrt{Z_{01}^{+} / Z_{10}^{+}} S_{22}^{12}+\sqrt{Z_{01}^{+} / Z_{10}^{-}} S_{12}^{12}\right)}{\left(Z_{01}^{+}+Z_{01}^{-}\right)} \\
& \alpha_{m m}^{y y}=\frac{a b}{2 j \omega} \frac{S_{11}^{22}+S_{22}^{22}-\sqrt{Z_{01}^{+} / Z_{01}^{-}} S_{21}^{22}-\sqrt{Z_{01}^{-} / Z_{01}^{+}} S_{12}^{22}-2}{\left(1 / Z_{01}^{+}+1 / Z_{01}^{-}\right)} \\
& \alpha_{m m}^{x y}=\frac{a b}{2 j \omega} \frac{\sqrt{Z_{10}^{+} / Z_{01}^{-}} S_{21}^{12}+\sqrt{Z_{10}^{-} / Z_{01}^{+}} S_{12}^{12}-\sqrt{Z_{10}^{-} / Z_{01}^{-}} S_{11}^{12}-\sqrt{Z_{10}^{+} / Z_{01}^{+}} S_{22}^{12}}{\left(1 / Z_{01}^{+}+1 / Z_{01}^{-}\right)} \\
& \alpha_{m e}^{y x}=-\frac{a b}{2 j \omega} \frac{\sqrt{Z_{01}^{-} Z_{01}^{+}} S_{21}^{22}-Z_{01}^{-} S_{11}^{22}+Z_{01}^{+} S_{22}^{22}-\sqrt{Z_{01}^{+} Z_{01}^{-}} S_{12}^{22}+Z_{01}^{-}-Z_{01}^{+}}{\left(Z_{01}^{+}+Z_{01}^{-}\right)} \\
& \alpha_{m e}^{x x}=\frac{a b}{2 j \omega} \frac{\sqrt{Z_{01}^{-} Z_{10}^{+}} S_{21}^{12}-\sqrt{Z_{01}^{-} Z_{10}^{-}} S_{11}^{12}+\sqrt{Z_{01}^{+} Z_{10}^{+}} S_{22}^{12}-\sqrt{Z_{01}^{+} Z_{10}^{-}} S_{12}^{12}}{\left(Z_{01}^{+}+Z_{01}^{-}\right)} \\
& \alpha_{e m}^{x y}=-\frac{a b}{2 j \omega} \frac{S_{21}^{22} / \sqrt{Z_{01}^{-} Z_{01}^{+}}+S_{11}^{22} / Z_{01}^{-}-S_{22}^{22} / Z_{01}^{+}-S_{12}^{22} / \sqrt{Z_{01}^{-} Z_{01}^{+}}-1 / Z_{01}^{-}+1 / Z_{01}^{+}}{\left(1 / Z_{01}^{+}+1 / Z_{01}^{-}\right)} \\
& \alpha_{e m}^{y y}=-\frac{a b}{2 j \omega} \frac{S_{21}^{12} / \sqrt{Z_{01}^{-} Z_{10}^{+}}+S_{11}^{12} / \sqrt{Z_{01}^{-} Z_{10}^{-}}-S_{22}^{12} / \sqrt{Z_{01}^{+} Z_{10}^{+}}-S_{12}^{12} / \sqrt{Z_{10}^{-} Z_{01}^{+}}}{\left(1 / Z_{01}^{+}+1 / Z_{01}^{-}\right)}
\end{aligned}
$$




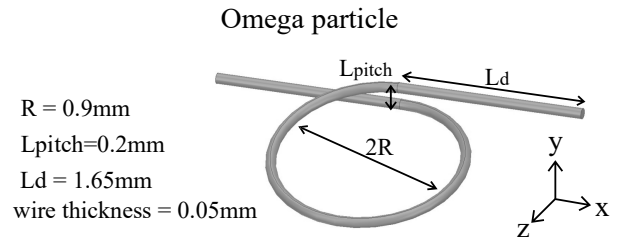

(a)

Magneto-dielectric sphere

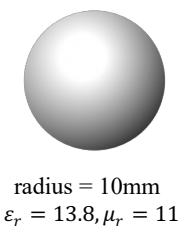

(b)

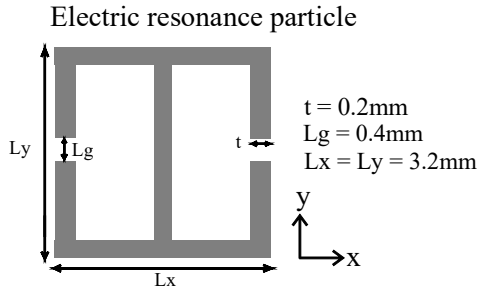

(c)

Figure 2: Particles schematics and dimensions and their associated local coordinate system. (a) Omega particle, (b) magneto-dielectric particle, and (c) an electric resonance particle.

\section{Results and Discussion}

In this section, we apply the proposed method to find the polarizability parameters of three different test scatterers by using their S-parameters, computed by the finite element method (FEM) of ANSYS HFSS 17 software.

As explained before, in the proposed WG-based method, there is a main approximation here; we assume effects of just two dominant (TE10 and TE01) modes and neglect those of evanescent modes in applying the BCs at the scatterer position. Furthermore, we assume smooth variations of the TE10 and TE01 mode amplitudes at about the scatterer $z$ position along $x$ and $y$ directions. This is done by choosing the size of the waveguide sides (relative to the length size of the scatterer) be large enough. A minimum length ratio about 5 is a proper value. To evaluate accuracy of the proposed method, two examples in a homogeneous medium including an omega particle and a magnetodielectric sphere and one example in half-space medium, an electric resonance particle placed on top of a half-space dielectric are simulated.

Here, for better comparisons, we normalize polarizability parameters. That is, $\alpha_{e e}, \alpha_{m m}$, and $\alpha_{e m / m e}$ are normalized respectively to $6 \pi \varepsilon_{0} / k_{0}^{3}, 6 \pi \mu_{0} / k_{0}^{3}$, and $6 \pi \varepsilon_{0} \eta_{0} / k_{0}^{3}$ Furthermore, in all examples the results are provided at about resonance frequencies. This has a practical importance because the numerical results are rapidly varying in these frequencies.

We used the methods in [8] and [3] to evaluate the presented method accuracy. These methods also use dipole model but without mentioned approximations of our proposed method. As we later see the results satisfy the Sipe-Kranendonk conditions [1].

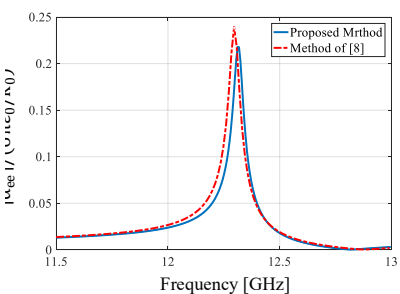

(a)

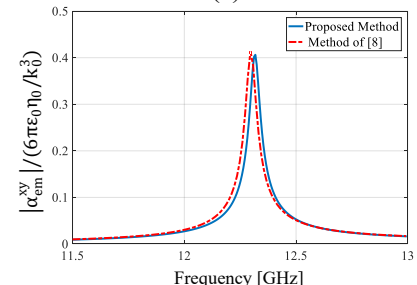

(c)

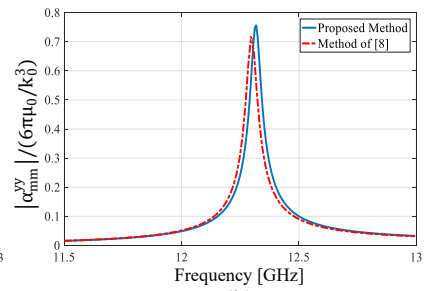

(b)

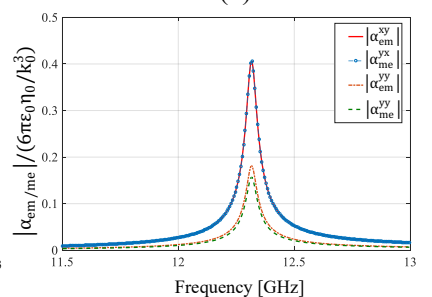

(d)
Figure 3: Normalized dominant polarizability parameters of the omega particle shown in Fig. 2 (a). We compared the results of the proposed waveguide-based method with those of the method explained in [8], summarized in Appendix B. (a) $\left|\alpha_{\mathrm{ee}}^{\mathrm{xx}}\right|$, (b) $\left|\alpha_{\mathrm{mm}}^{\mathrm{yy}}\right|$, and (c) $\left|\alpha_{\mathrm{em}}^{\mathrm{xy}}\right|$. In (d), the reciprocity (i.e., $\left|\alpha_{\mathrm{em}}^{\mathrm{xy}}\right|=\left|\alpha_{\mathrm{me}}^{\mathrm{yx}}\right|$ and $\left|\alpha_{\mathrm{em}}^{\mathrm{yy}}\right|=\left|\alpha_{\mathrm{me}}^{\mathrm{yy}}\right|$ ) are demonstrated and two bi-anisotropies (i.e., $\left|\alpha_{\mathrm{em}}^{\mathrm{xy}}\right|>\left|\alpha_{\mathrm{em}}^{\mathrm{yy}}\right|$ ) are compared.

\subsection{Omega particle}

An omega particle is shown in Fig. 2 (a). This particle produces omega-coupling type bi-anisotropy and because of its non-planar (volumetric) structure, some chirality is also expected. As shown in the Fig. 2 (a), this scatterer produces dominant $x$-directed electric polarizability $\left(\alpha_{\mathrm{ee}}^{\mathrm{xx}}\right), y$-directed magnetic polarizability $\left(\alpha_{\mathrm{mm}}^{\mathrm{yy}}\right)$ and magneto-electric coupling $\left(\alpha_{\mathrm{em}}^{\mathrm{xy}}\right)$ (which is equal to negative of $\alpha_{\mathrm{me}}^{\mathrm{yx}}$ due to the reciprocity). All of these polarizabilities can be determined by one simulation once the particle is properly placed inside the WG.

Let us look for the polarizability parameters within the frequency range of $12-13 \mathrm{GHz}$ (i.e., inside the $\mathrm{X}$ band frequencies), once the particle is in the free-space. As shown in Fig. 2 (a), the scatterer maximum length is $3.3 \mathrm{~mm}$, about one seventh of the maximum wavelength $(\lambda=2.5 \mathrm{~mm}$ at 12 $\mathrm{GHz}$ ). The scatterer is small enough to meet the dipole approximation requirement. We set dimensions $a=$ $16.5 \mathrm{~mm}$ and $b=15 \mathrm{~mm}$ (see Fig. 1) to make TE10 and TE01 modes propagate inside the WG at the minimum frequency point $12 \mathrm{GHz}$ and prevent higher-order modes propagation in the desired frequency range 12-13 GHz. Ports are at $z= \pm h$ where $h=50 \mathrm{~mm}$, is set long enough so that higher-order mode effects could be suppressed. Simulation results are presented in Fig. 3 and compared with those of the method presented in [8]. The method [8] is for particles in homogeneous media. Important relations of [8] are provided in Appendix II, for completeness.

According to Fig. 3 (a) to (c), waveguide method results are in good agreement with the results obtained from freespace method of [8], where its only approximation is 


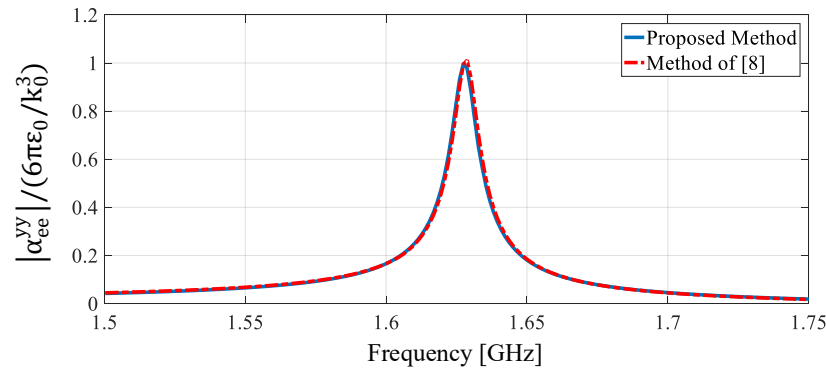

(a)

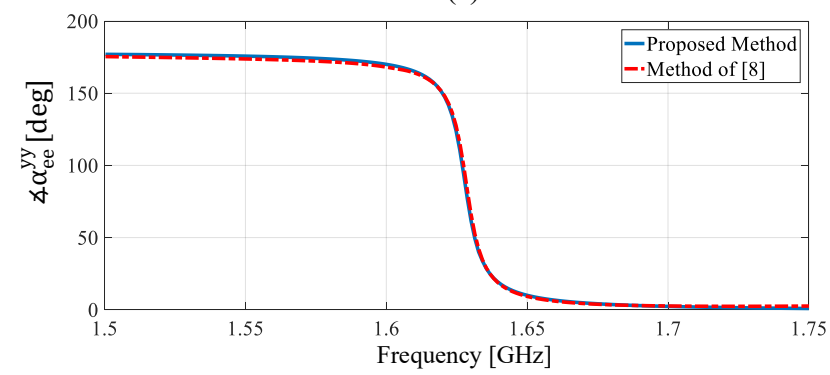

(b)

Figure 4: Magnitude and phase of normalized electric polarizability parameter of magneto-dielectric sphere. The results are compared with the free-space method explained in [8], summarized in Appendix II.

consideration of dipole response and ignoring multipole effects. Fig. 3 (d) compares different types of bianisotropies, where as expected omega-coupling type is the dominant one (i.e., $\left|\alpha_{\mathrm{em}}^{\mathrm{xy}}\right|>\left|\alpha_{\mathrm{em}}^{\mathrm{yy}}\right|$ ). Here, we have also observed (but not shown) that the reciprocity is met; $\alpha_{e m}^{x y}=$ $-\alpha_{m e}^{y x}$ and $\alpha_{e m}^{y y}=-\alpha_{m e}^{y y}$ [1]. The Sipe-Kranendonk conditions is satisfied (imaginary part of inversed electric polarizability of the lossless particle is $k_{0}^{3} / 6 \pi \varepsilon_{0}$ ).

The presented method works almost accurate as the method in [8], while it uses considerably simpler configuration for the most general bi-anisotropic example with transverse polarizabilities.

\subsection{Magneto-dielectric sphere}

Magneto-dielectric sphere provides isotropic electric and magnetic polarizabilities. By parameters given in Fig. 2 (b), the resonance frequency occurs at about $1.63 \mathrm{GHz}$. Therefore, maximum size of the particle $(2 \mathrm{~cm})$ is about $1 / 9$ of the free-space wavelength at resonance frequency. This example is an ideal small scatterer, accurately modeled by dipole moments. The dipole approximation is more justified comparing to the previous example (Sec. 4.1). As shown in Fig. 4, excellent agreement and more accurate results are obtained for the magneto-dielectric sphere. The normalized polarizability amplitude in Fig. 4 (a) is equal to 1 , as expected from the Sipe-Kranendonk conditions.

Here, in order to operate at the minimum frequency of $1.5 \mathrm{GHz}$ we set the waveguide dimensions $a=165 \mathrm{~mm}$ and $b=150 \mathrm{~m}$. The waveguide length is $2 h$ where $h=$ $500 \mathrm{~mm}$. Simulation results are presented in Fig. 4 and compared with the results of the method presented in [8].

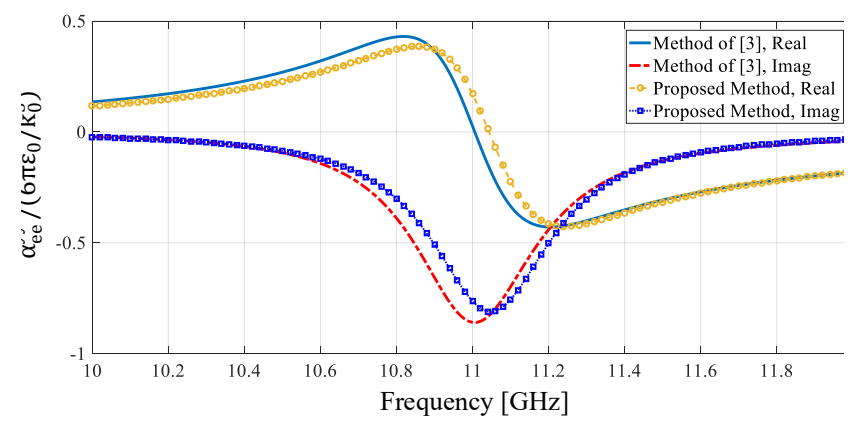

Figure 5: Real and imaginary parts of normalized $y$ directed electric polarizability of electric resonance particle. The results are compared with the method explained in [3], reviewed in Appendix III.

\subsection{Electric Resonance Particle}

In practice particles are on top of a dielectric medium or on a slab. For low relative permittivity values, our WGbased method is still an appropriate and easy-to-use tool that provides fast and yet accurate results for general cases where bi-anisotropic dipole scatterers are considered.

Here, as an example we study a scatterer shown in Fig. 2 (c) placed above a half-space dielectric medium with relative permittivity of $\varepsilon_{r}=2.2$. This particle provides dominant electric polarizability in the $y$-direction $\left(\alpha_{\mathrm{ee}}^{\mathrm{yy}}\right)$. The results of the proposed waveguide method are compared with the method of [3] (which is for particles above halfspace and explained in Appendix III) and presented in Fig. 5 , where good agreement is shown. The Sipe-Kranendonk conditions is satisfied. The absolute value of the normalized polarizability at the resonance frequency is slightly less than 1 because the effective medium has permittivity more than $\varepsilon_{0}$.

In this simulation, the waveguide parameters are $a=$ $18.7 \mathrm{~mm}, b=17 \mathrm{~mm}$, and $h=70 \mathrm{~mm}$. It should be noted that the results do not have noticeable changes for different choices of waveguide sides as long as only the first dominant modes TE01 and TE10 are excited and higherorder modes effects could be negligible.

Method of [3] is the most general method presented in the literature, however, requires integration of currents over the scatterer and incidence with different angles and polarizations. In comparison, our WG-based proposed method is considerably simple and provide fast simulation runtime with an acceptable accurate results. However, it is restricted to transverse polarizability extraction.

\section{Conclusions}

In this paper, we provided general and explicit formulations for polarizabilities of a transversal dipole scatterer above half-space in Eqs. (10) and (11). The method is based on the S-parameters of a rectangular waveguide. We presented detailed formulations for the general case in which the waveguide is half filled by a dielectric. The final relations are explicitly given in terms of the generalized scattering parameters. The method main approximation is in 
applying the boundary condition at the place of the scatterer where only TE10 and TE01 modes are assumed to have dominant contributions. That means we neglected the minor effects of higher order waveguide modes. The numerical examples showed that this approximation is valid and still accurate results are obtained. The method was applied to three different examples, including a bi-anisotropic omega particle and a magneto-dielectric sphere both placed inside the free-space and an electric resonance particle placed on top of a half-space dielectric medium. They are numerically challenging and practically important problems. Acceptable accuracies in all cases are observed. The method main advantages are being fast, easy to use, general, and yet accurate for transversal dipole scatterers.

\section{Appendix I}

In this appendix, we explain calculation of $p \frac{ \pm}{y}$ for TE10 incidence given in (8) to clarify the derivation process.

Using the boundary condition (2), $p_{y}^{+}$and $p_{y}^{-}$are respectively induced by TE10 incidences from port 1 (in $\mathrm{z}<0$ ) and port 2 (in $\mathrm{z}>0$ ) and are related to the $x$-directed difference magnetic fields as follow.

$j \omega p_{y}^{+} \delta(x-a / 2) \delta(y-b / 2)=H_{x}\left(z=0^{+}\right)-H_{x}\left(z=0^{-}\right)$,

$H_{x}\left(z=0^{+}\right)=H_{x \mathrm{sc}}^{+}, H_{x}\left(z=0^{-}\right)=H_{x \mathrm{sc}}^{-}+H_{x \mathrm{inc}}^{-}$,

$j \omega p_{y}^{-} \delta(x-a / 2) \delta(y-b / 2)=H_{x}\left(z=0^{+}\right)-H_{x}\left(z=0^{-}\right)$,

$H_{x}\left(z=0^{+}\right)=H_{x \mathrm{inc}}^{+}+H_{x \mathrm{sc}}^{+}, H_{x}\left(z=0^{-}\right)=H_{x \mathrm{sc}}^{-}$,

where $H_{x s c}^{ \pm}$is the TE10 scattered field in $z>0$ and $z<0$ regions and $H_{\text {xinc }}^{-}\left(H_{\text {xinc }}^{+}\right)$is the incident magnetic field of port1 (port2) in region $z<0(z>0)$.

Using the scattered fields (7), $H_{x s c}^{+}=-\frac{B_{10}^{+}}{Z_{10}^{+}}, H_{x s c}^{-}=+\frac{B_{10}^{-}}{Z_{10}^{-}}$ and employing $H_{x i n c}^{ \pm}= \pm \frac{E_{0}}{z_{10}^{ \pm}}$, and then taking the inner product of both sides of A.1 by $\sin \left(\frac{\pi x}{a}\right)$ (i.e., $\left.\int_{0}^{a} \int_{0}^{b}(\ldots) \sin \left(\frac{\pi x}{a}\right) d x d y\right)$, we end up with a relation that expresses $p_{y}^{ \pm}$in terms of the scattered field amplitudes as follow,

$$
p_{y}^{ \pm}=\frac{a b}{2 j \omega}\left(-\frac{B_{10}^{+}}{Z_{10}^{+}}-\frac{B_{10}^{-}}{Z_{10}^{-}}+\frac{E_{0}}{Z_{10}^{ \pm}}\right) .
$$

\section{Appendix II}

In this appendix, we provide relevant relations from [8], employed here to validate results of the first (omega particle) and the second (magneto-dielectric particle) examples. This method works for a particle in a homogenous medium and similar to the method in [3] uses plane wave illuminations. To obtain induced dipole moments, using dipole model, the scattered electric and magnetic fields are measured in the far-field region at $z=$ $\pm h$. Combining the sampled scattered fields from four different setups $( \pm z$ ward incidences with two $x$ and $y$ directed polarizations), the particle transverse to $z$ polarizabilities are extracted. In summary, we have

$$
\begin{aligned}
& \alpha_{e e}^{x x}=\frac{1}{4 \gamma E_{0}}\left[{ }_{+h} E_{x}^{+}+{ }_{-h} E_{x}^{+}+{ }_{-h} E_{x}^{-}+{ }_{+h} E_{x}^{-}\right], \\
& \alpha_{m m}^{y y}=\frac{\eta^{2}}{4 \gamma E_{0}}\left[{ }_{+h} E_{x}^{+}+{ }_{-h} E_{x}^{-}-{ }_{+h} E_{x}^{-}-{ }_{{ }_{h}} E_{x}^{+}\right], \\
& \alpha_{e m}^{x y}=\frac{\eta}{4 \gamma E_{0}}\left[{ }_{+h} E_{x}^{+}+{ }_{-h} E_{x}^{+}-{ }_{-h} E_{x}^{-}-{ }_{+h} E_{x}^{-}\right] .
\end{aligned}
$$

where $\gamma=\frac{k^{2}}{4 \pi \varepsilon_{0}} e^{-j k h}, k$ is the wave number in the host medium, and ${ }_{ \pm h} E_{\bar{x}}^{+}$is the $x$-polarized scattered field measured at $z= \pm h$ (specified by the left-subscript), illuminated by a $( \pm) z$-ward incident field (specified by the superscript).

\section{Appendix III}

In this appendix, we provide a review of important relations from [3], employed here to validate results of our WG-based method for the third example (Fig. 2 (c) and Sec. 3.3). This method is based on plane wave illuminations (at different directions and for various polarizations) of a scatterer in an unbounded half-space medium. First, induced electric $(\bar{p})$ and magnetic $(\bar{m})$ dipole moments are obtained by integrating the currents of the scatterer as follow,

$$
\begin{gathered}
\bar{p}=\frac{1}{j \omega} \int_{V} \bar{J}(\bar{r}) d v, \\
\bar{m}=\frac{1}{2} \int_{V} \bar{r} \times \bar{J}(\bar{r}) d v,
\end{gathered}
$$

where $V$ is the volume of the scatterer, $\bar{r}$ is the position vector in the Cartesian coordinate system and $\bar{J}(\bar{r})$ is the volume electric current density, induced in the scatterer due to a plane-wave illumination. Next, the electric and magnetic polarizabilities are computed [3]. Here, for example the electric polarizability $\left(\alpha_{e e}^{y y}\right)$ retrieved using numerically evaluated dipole moments $p_{y}^{+}$and $p_{y}^{-}$is given by

$$
\alpha_{e e}^{y y}=\frac{Z^{-} p_{y}^{+}+Z^{+} p_{y}^{-}}{\left(Z^{-}+Z^{+}\right) E_{0}}
$$

where $Z^{-}$and $Z^{+}$are characteristic impedances of $z<0$ and $z>0$ regions, respectively. The $p_{y}^{ \pm}$is obtained by forward and backward (along $+z$ and $-z$ directions) illuminations and $E_{0}$ is the $y$-directed incident electric field amplitude. It is noted that for the electric resonance particle assumed in the Fig. 2 (c), transverse magnetic polarizabilities are negligible.

\section{References}

[1] S. Tretyakov, Analytical modeling in applied electromagnetics. Artech House, 2003.

[2] X.-X. Liu, Y. Zhao, and A. Alu, "Polarizability Tensor Retrieval for Subwavelength Particles of Arbitrary Shape," IEEE Trans. Antennas Propag., vol. 64, no. 6, pp. 2301-2310, Jun. 2016.

[3] M. Yazdi and N. Komjani, "Polarizability calculation of arbitrary individual scatterers, scatterers in arrays, and substrated scatterers," J. Opt. Soc. Am. B, vol. 33, no. 3, p. 491, Mar. 2016.

[4] S. A. Tretyakov, S. Maslovski, and P. A. Belov, "An analytical model of metamaterials based on loaded wire 
dipoles," IEEE Trans. Antennas Propag., vol. 51, no. 10, pp. 2652-2658, Oct. 2003.

[5] S. A. Tretyakov, F. Mariotte, C. R. Simovski, T. G. Kharina, and J.-P. Heliot, "Analytical antenna model for chiral scatterers: comparison with numerical and experimental data," IEEE Trans. Antennas Propag., vol. 44, no. 7, pp. 1006-1014, Jul. 1996.

[6] C. R. Simovski, S. A. Tretyakov, A. A. Sochava, B. Sauviac, F. Mariotte, and T. G. Kharina, "Antenna Model for Conductive Omega Particles," J. Electromagn. Waves Appl., vol. 11, no. 11, pp. 15091530, Jan. 1997.

[7] R. Alaee et al., "Magnetoelectric coupling in nonidentical plasmonic nanoparticles: Theory and applications," Phys. Rev. B, vol. 91, no. 11, p. 115119, Mar. 2015.

[8] V. S. Asadchy, I. A. Faniayeu, Y. Ra'di, and S. A. Tretyakov, "Determining polarizability tensors for an arbitrary small electromagnetic scatterer," Photonics Nanostructures - Fundam. Appl., vol. 12, no. 4, pp. 298-304, Aug. 2014.

[9] A. D. Scher and E. F. Kuester, "Extracting the bulk effective parameters of a metamaterial via the scattering from a single planar array of particles," Metamaterials, vol. 3, no. 1, pp. 44-55, Mar. 2009.

[10] T. D. Karamanos, A. I. Dimitriadis, and K. Nikolaos V., "Polarizability Matrix Extraction of a Bianisotropic Metamaterial from the Scattering Parameters of Normally Incident Plane Waves," Adv. Electromagn., vol. 1, no. 3, p. 64, Nov. 2012.

[11] S. A. Tretyakov, S. I. Maslovski, I. S. Nedvedov, A. J. Vitanen, P. A. Belov, and A. Sanmartin, "Artificial Tellegen Particle," Electromagnetics, vol. 23, no. 8, pp. 665-680, Jan. 2003.

[12] E. F. Kuester, M. A. Mohamed, M. Piket-May, and C. L. Holloway, "Averaged transition conditions for electromagnetic fields at a metafilm," IEEE Trans. Antennas Propag., vol. 51, no. 10, pp. 2641-2651, Oct. 2003.

[13] R. F. Harrington, Time-harmonic electromagnetic fields. IEEE Press, 2001.

[14] M. Albooyeh, R. Alaee, C. Rockstuhl, and C. Simovski, "Revisiting substrate-induced bianisotropy in metasurfaces," Phys. Rev. B, vol. 91, no. 19, p. 195304, May 2015.

[15] D. M. Pozar, Microwave Engineering. John Wiley \& Sons, 2012. 